\title{
Dynamics of TBP binding to the TATA box
}

\author{
Peter Schluesche ${ }^{\mathrm{a}}$, Gregor Heiss ${ }^{\mathrm{a}}$, Michael Meisterernst ${ }^{\mathrm{bc}}$ Don C. Lamb*ad \\ ${ }^{a}$ Physical Chemistry, Dept of Chemistry and Biochemistry and Center for NanoScience, Ludwig- \\ Maximilians-Universität München, Butenandtstrasse 11, 81377 Munich, Germany. \\ ${ }^{\mathrm{b}}$ Helmholtz Center Munich German Research Center for Environmental Health, Gene Expression \\ Marchionini-Str. 25, 81377 Munich, Germany. \\ 'Institute for Cell and Tumor Biology, Department of Medicine, Westfalian Wilhelms University, \\ 48149 Muenster, Germany. \\ ${ }^{\mathrm{d}}$ Dept. of Physics, University of Illinois at Urbana-Champaign, 1110 W. Green St., Urbana, IL \\ 61801, USA.
}

\begin{abstract}
Gene expression is highly controlled and regulated in living cells. One of the first steps in gene transcription is recognition of the promoter site by the TATA box Binding Protein (TBP). TBP recruits other transcriptions factors and eventually the RNA polymerase II to transcribe the DNA in mRNA. We developed a single pair Förster Resonance Energy Transfer (spFRET) assay to investigate the mechanism of gene regulation. Here, we apply this assay to investigate the initial binding process of TBP to the adenovirus major late (AdML) promoter site. From the spFRET measurements, we were able to identify two conformations of the TBP-DNA complex that correspond to TBP bound in the correct and the opposite orientation. Increased incubation times or the presence of the transcription factor TFIIA improved the alignment of TBP on the promoter site. Binding of TBP to the TATA box shows a rich dynamics with abrupt transitions between multiple FRET states. A frame-wise histogram analysis revealed the presence of at least six discrete states, showing that TBP binding is more complicated than previously thought. Hence, the spFRET assay is very sensitive to the conformation of the TBP-DNA complex and is very promising tool for investigating the pathway of TBP binding in detail.
\end{abstract}

Keywords: Single Molecule Microscopy, Single Pair Förster Resonance Energy Transfer, Transcription, TATA Box Binding Protein

\section{INTRODUCTION}

DNA is the guardian of genetic code. To convert the genetic information into a functional protein, DNA must first be transcribed into RNA. One of the first steps in the transcription process in eukaryotic cells is recognition of the promoter site of the gene that is to be transcribed. This occurs via the binding of the TATA box Binding Protein (TBP) to the promoter site. TBP then recruits other transcription factors and eventually RNA polymerase II to form the preinitiation complex. As initiation of transcription is an early step in gene expression, it is a popular target for gene regulation and control.

We have investigated the mechanism of gene regulation using single-pair (sp) FRET between TBP and DNA containing the AdML TATA promoter ${ }^{1}$. A donor fluorophore was attached specifically to recombinant yeast TBP (at position 61) and the acceptor molecule was bound to the DNA. The residue and base to which the donor and acceptor fluorophores are attached are shown as green and red space filled structures respectively in Fig. 1. After incubating the TBP and DNA at $28^{\circ} \mathrm{C}$ for approximately 15 minutes, a stable TBP-DNA complex was formed. Nonstable complexes were washed away and more than $90 \%$ of the complexes demonstrated a steady FRET signal when immobilized on the surface. Upon addition of the transcription cofactor NC2 (also known as Dr1-DRAP) ${ }^{2,3}$ to the sample chamber, more than $75 \%$ the TBP-DNA complexes demonstrated dynamic fluctuations in its conformation which can lead to movement of TBP-NC2 along the DNA. These studies revealed a novel function for NC2. Here, we use the same spFRET assay to investigate the binding of TBP to the TATA box of the AdML promoter.

Single Molecule Spectroscopy and Imaging

edited by Jörg Enderlein, Zygmunt K. Gryczynski, Rainer Erdmann

Proc. of SPIE Vol. 6862, 68620E, (2008) · 1605-7422/08/\$18 - doi: 10.1117/12.769177

Proc. of SPIE Vol. $686268620 \mathrm{E}-1$ 
According to the crystal structure of TBP bound to DNA containing the adenovirus major late (AdML) promoter, the DNA is strongly deformed (Fig. 1). The TBP has two pairs of phenylalanine resides intercalate into the minor grove on both sides of the TATA box. This leads to a widening of the minor grove, a compaction of the major grove, and a $117^{\circ}$ unwinding of the DNA helix ${ }^{4}$. The mechanism and the kinetics of TBP binding has been investigated extensively by Parkhurst and coworkers ${ }^{5-8}$. Using stopped flow mixing and ensemble Förster Resonance Energy Transfer (FRET) experiments, they have established the existence of two intermediate states in the TBP binding process to the AdML and E4 promoter sites. A larger number of intermediate states have been observed in the binding of TBP binding to other promoter sites ${ }^{8}$. The model for TBP binding to the AdML promoter is shown schematically in Fig 2.
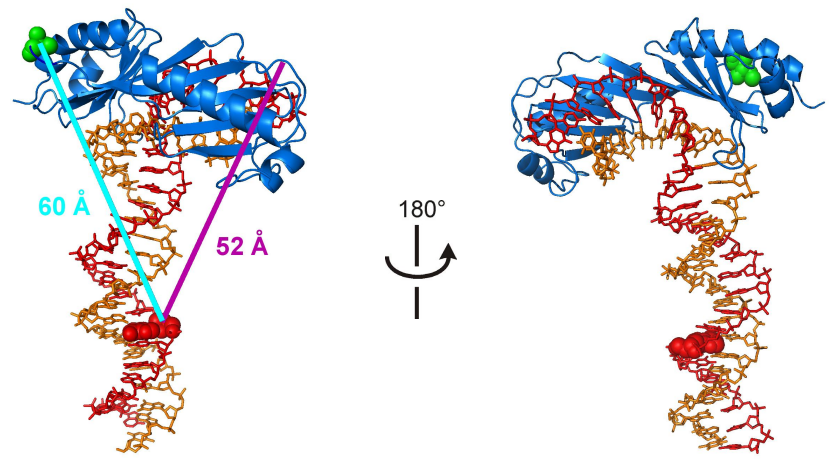

Fig. 1. Structure of the TBP-DNA complex. The crystal structure of the TBP-DNA complex (PDB structure 1RM1) is shown from two angles. The DNA from the PDB file was extended upstream assuming B-form DNA to show the labeling position of the acceptor. TBP is shown in blue and the DNA in red and orange. The residue and base to which the donor and acceptor fluorophores are attached are shown as space filling structures in green and red respectively. A large deformation of the DNA is observed in the TBP bound complex. The approximate distance between the attachment points for TBP in the correct orientation (light blue) and the opposite orientation (violet) are given.

The model of Parkhurst has recently been supported by Tolić-Nørrelykke and coworkers using single molecule measurements ${ }^{9}$. Different conformations of the DNA were observed by monitoring the Brownian motion of tethered beads using video microscopy and by optical tweezers experiments. Polystyrene beads were attached to one end of the DNA while the opposite end was bound to the surface. The motion of the tethered-particle changes when the flexibility or length of the DNA changes. Three states were visible corresponding to high, intermediate and low mobility of the beads. The high mobility state corresponds to the DNA in the absence of TBP. The first intermediate was assigned to the first pair of phenylalanine residues intercalating on the 5' end of the TATA box. It has previously been shown that the DNA bending occurs simultaneously with TBP binding ${ }^{5}$. In the first intermediate state, it is thought that the transcription factor, TFIIA can bind. The second intermediate state is formed when the second phenylalanine pair intercalates into the other side of the TATA box. The final state is formed upon binding of the transcription factor TFIIB to the complex.

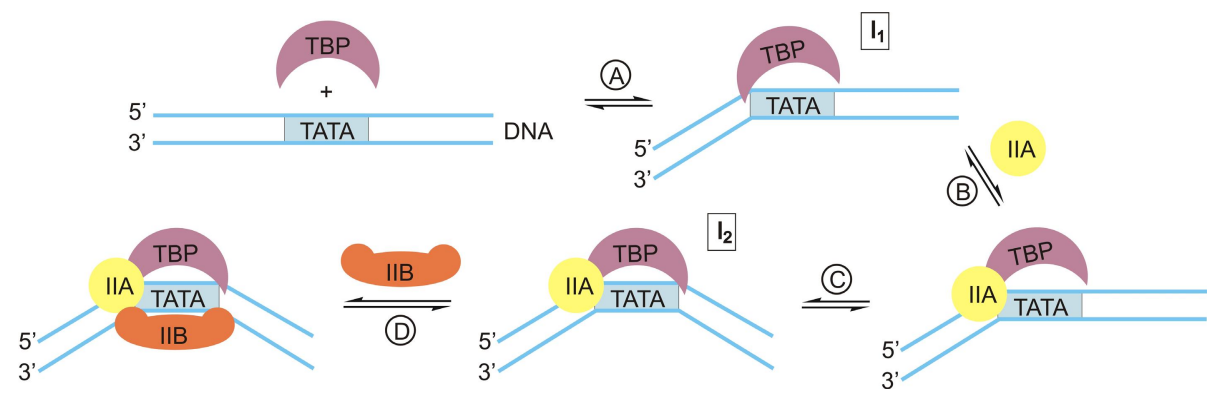

Fig. 2. Model of TBP binding. Two intermediate states are observed upon TBP binding to DNA. IN the first intermediate state, one pair of phenylalanine residues intercalate into the minor grove of the DNA. The transcription factor TFIIA is thought to bind to this configuration. In the second intermediate state, the second pair of phenylalanine residues intercalates into the DNA on the opposite side of the TATA box. 


\section{METHODOLOGY}

For the experiments presented here, we used TBP from yeast with Cys - Ala mutations at positions 78 and 164 and a Ser - Cys mutation at position 61, kindly provided by Tony Weil. The protein was specifically labeled at position 61 with Atto532. The labeling procedure is described in detail in the supplementary materials of Schluesche et $\mathrm{al}^{1}$. The label is optimally positioned at the boundary of the highly conserved region of TBP and the was shown not to interfere with the functionality of the protein ${ }^{10,11}$.

A 70 base pair DNA fragment from the AdML promoter was synthesized and labeled with Atto647N attached to a thymine base at the position highlighted below in red:

$$
\begin{aligned}
& 5 \text { ' - . ... GGGTGTTCCTGAAGGGGGGC TATAAAA GGGGGTGGGGGCGCGTTCG... . T-Biotin } \\
& 3 \text { ' - . . . CCCACAAGGACTTCCCCCCGATATTTTCCCCCACCCCCGCGCAAGC.. . A }
\end{aligned}
$$

The DNA was purified using preparative HPLC and PAGE assays (native SDS-gel). Experiments were performed in working buffer. Working buffer consists of $12 \mathrm{mM}$ Tris- $\mathrm{HCl} \mathrm{pH} 7.3,60 \mathrm{mM} \mathrm{KCl}, 4 \mathrm{mM} \mathrm{MgCl} 2,25 \mathrm{mM}$ Hepes- $\mathrm{KOH}$ $\mathrm{pH} 8.2,12 \%$ (v/v) glycerol, $0.25 \mathrm{mg} \mathrm{ml}-1$ BSA, $0.6 \mathrm{mM}$ EDTA, $2.5 \mathrm{mM}$ DTT, $0.5 \mathrm{mM}$ PMSF and 0.025\% NP-40 dissolved in Millipore water.

\subsection{Total Internal Reflection Fluorescence Microscopy}

Experiments were performed on a home built prism-type Total Internal Reflection Fluorescence Microscope (TIRFM). Details of the setup can be found in Schluesche et $\mathrm{al}^{1}$. The system was equipped with $532 \mathrm{~nm}$ (Nd:YAG Laser) and $633 \mathrm{~nm}$ (HeNe Laser) excitation and the excitation wavelength was controlled with shutters and a homebuilt shutter controller. Hence, measurements could be performed using millisecond Alternating Laser Excitation (msALEX) ${ }^{12}$ where alternating frames could be collected with $532 \mathrm{~nm}$ excitation and $633 \mathrm{~nm}$ excitation respectively. By alternating the excitation wavelength frame by frame, we could monitor the photodynamics of the acceptor in parallel to the FRET signal to ensure that the observed dynamics was not due to photoblinking of the acceptor. Shortly before the excitation beam enters the prism, the laser beam is circularly polarized with the aid of a $\lambda / 4$ plate. The angle that the incoming excitation beam makes with the bottom of the prism was $72^{\circ}$. The intensity at the total-internal-reflection interface was $350-670 \mathrm{nW} / \mu \mathrm{m}^{2}$. Fluorescence emitted from dye molecules in the evanescent field was collected with a $1.2 \mathrm{NA}$ water immersion objective. The fluorescence emission spectrum was split into donor and acceptor emission channels and focused onto different regions of an EMCCD camera. The imaged area was $35 \times 70 \mu \mathrm{m}^{2}$ for each channel. Data was collected with a temporal resolution of $75 \mathrm{~ms} /$ frame.

The sample was attached directly to the quartz prisms used for the TIRFM measurements. The quartz surface was silanized and coated with polyethylene glycol (PEG) to hinder the absorption of TBP to the prism surface. DNA was attached to a small fraction of biotinulated PEG via a biotin-streptavidin-biotin linkage. Two holes were drilled into the quartz prism and a flow cell was built by sandwiching a piece of Nescofilm between the prism and the coverslip. A channel was cut in the Nescofilm connecting the two holes drilled through the prism. Hence, different buffers could be flown through the sample chamber during the experiment. TBP-DNA complexes were prepared for single-molecule studies by either forming the complexes directly in the sample chamber at concentrations appropriate for single-molecule experiments or by preincubating the sample at higher concentration and diluting it to single-molecule concentrations directly before adding it to the sample chamber. For experiments investigating the orientation of TBP on the TATA Box, excess complexes were removed before the data were collected. In the TBP binding studies, excess TBP is necessary and was not removed from the sample chamber.

\subsection{Single pair FRET}

The FRET efficiency, $E$, of individual complexes was determined from the fluorescence intensities of the donor and acceptor channels as shown in eq (1):

$$
E=\frac{I_{A}}{\alpha I_{D}+I_{A}}
$$

where $I_{D}$ and $I_{A}$ are the fluorescence intensity of the donor and acceptor channels respectively and $\alpha$ is the detection correction factor, which corrects for the differences in sensitivity of the apparatus to the two fluorophores. The 
fluorescence intensity of the donor and acceptor channels were automatically determined using a home-written analysis routine in Matlab. The fluorescence intensity was calculated from the total intensity within a box of $7 \times 7$ pixels surrounding the selected particle minus the local background determined in a region of $8 \times 8$ to $11 \times 11$ pixels about the selected particle.

The resulting FRET traces from individual complexes were analyzed manually using a self-written routine programmed in PvWave. The contributions from spectral cross-talk of the donor into the acceptor channel and from direct excitation of the acceptor molecule were negligible and not corrected for in the analysis. Determination of the detection correction factor, $\alpha$, plays an important role in the quality of the FRET histograms determined from the single molecule data. $\alpha$ varies widely from molecule to molecule and is best determined separately for each individual complex. The detection correction factor can be determined from the change in donor and acceptor intensities upon acceptor photobeaching. In the experiments presented here, the acceptor rarely bleaches before the donor, making an individual $\alpha$ analysis impossible. As the average $\alpha$ varies with the experimental parameters such as the focus and correction collar setting of the objective, we determined $\alpha$ separately for each experiment. Variations in $\alpha$ ranged from 0.60 to 0.70 .

\section{ORIENTATION OF TBP ON THE TATA BOX}

\subsection{Results}

TBP is a very symmetric molecule (Fig. 1). Although it is only observed in one orientation in x-ray structures, Schepartz and coworkers have shown that TBP can bind to the TATA box in two orientations ${ }^{13-15}$. The direction of Polymerase II translation along the DNA is determined by the orientation of $\mathrm{TBP}^{16}$ on the TATA box. Therefore, general transcription factors play an important role in stabilizing the correct orientation of TBP on DNA ${ }^{14}$.

We investigated the orientation of TBP on the TATA box using spFRET measurements. For these experiments, the surfaced of the sample chamber was sparsely coated with DNA containing the AdML promoter site. Excess DNA was removed from the sample chamber and $500 \mathrm{pM}$ TBP was added to the sample chamber. TBP was allowed to react with the DNA for 10 minutes, after which free TBP and TBP that had not tightly bound to the DNA was removed by flushing with working buffer.
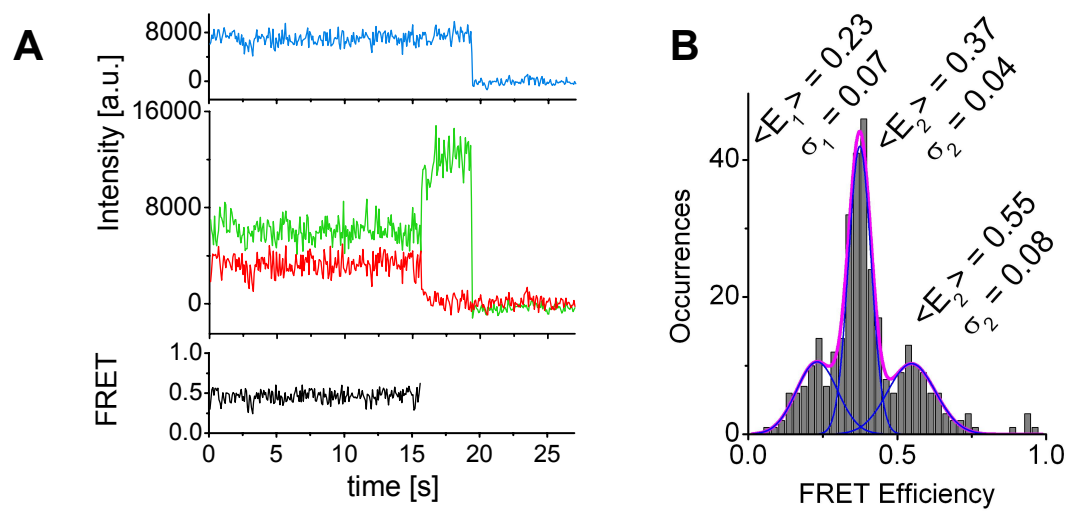

Fig. 3: SpFRET measurements of the stable TBP-DNA complex. A) The total intensity $\left(I_{T}=\alpha I_{A}+I_{D}\right)$ (in blue), the donor intensity (in green), the acceptor intensity (in red) and the FRET efficiency (in black) of a single TBP-DNA complex are shown as a function of time. After 16 seconds, the acceptor molecule photobleaches and the donor molecule fluoresces at full intensity until it photobleaches three seconds later. B) A histogram of average FRET efficiencies determined from 323 single TBP-DNA complexes.

A spFRET trace of a TBP-DNA complex is shown in Fig. 3A. A steady FRET signal is observed for approximately 16 seconds before the acceptor molecule photobleaches. As the FRET efficiency is constant over the trace, we can average the FRET efficiencies from each frame to provide an accurate average FRET efficiency for the individual complexes. Fig. 3B shows a histogram of the average FRET efficiency from 323 molecules. Three peaks are clearly observable in the histogram of FRET efficiencies with peak FRET efficiencies of $23 \%, 37 \%$, and $55 \%$. To increase the subpopulation of correctly orientated TBP molecules, $10 \mathrm{nM}$ TBP was preincubated with $10 \mathrm{nM}$ DNA for 30 minutes in the presence 
and absence of $20 \mathrm{nM}$ TFIIA before being diluted to single-molecule concentrations and immobilized on the prism surface. The biotinulated complexes were allowed to react for 10 minutes with the steptavidin prepared surface before excess complexes were removed from solution. Histograms of spFRET efficiencies are shown in Fig 4B and 4C respectively. The subpopulations at $\sim 20 \%$ and $\sim 40 \%$ subpopulations are still present but the subpopulation at $55 \%$ FRET efficiency is suppressed with preincubation or with the addition of TFIIA. From our previous work, it is clear that the $40 \%$ FRET peak corresponds to TBP bound with the correct orientation on the TATA box ${ }^{1}$. The origin of the $20 \%$ FRET peak is still unknown most likely arises from TBP binding at a different location on the DNA.
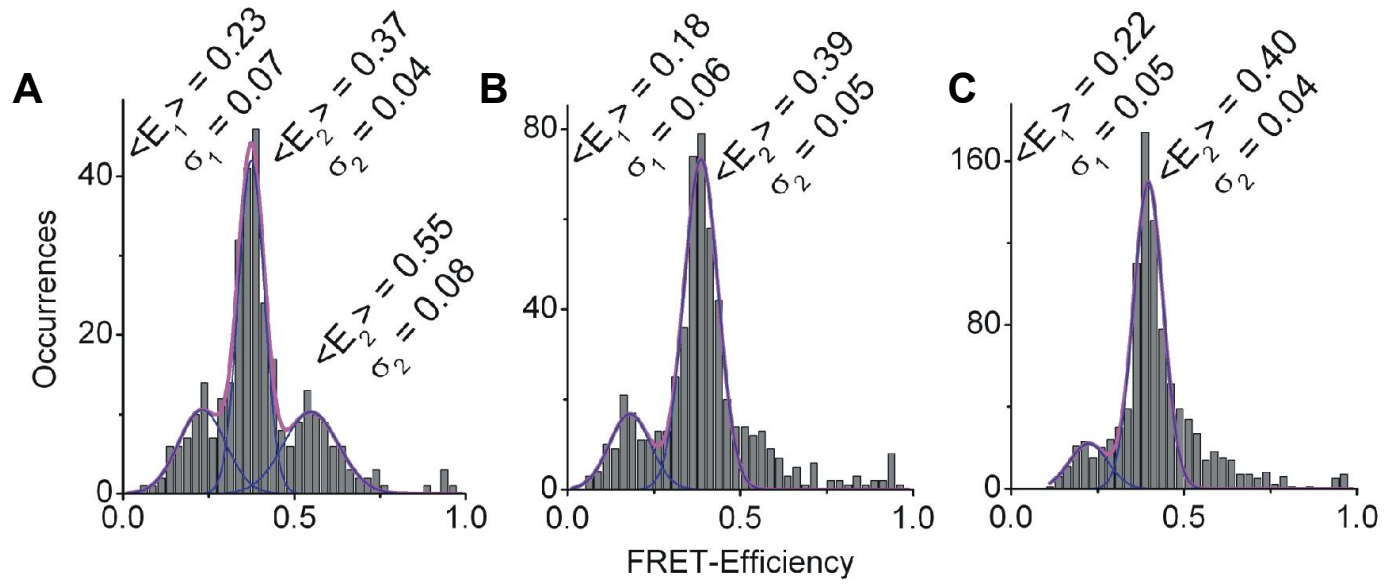

Fig. 4. Histograms of spFRET efficiencies for TBP-DNA complexes. A) Complexes $(\mathrm{N}=323)$ that were formed directly on the surface of the sample chamber, B) complexes $(\mathrm{N}=552)$ formed by preincubation of TBP and DNA before addition to the sample chamber and $\mathrm{C})$ complexes $(\mathrm{N}=944)$ formed as in B with a 2 fold excess of TFIIA.

\subsection{Discussion}

Schepartz and coworkers have shown in elegant cleavage experiments that TBP can bind with two orientations on the TATA box. They attached a phenanthroline reagent to a mutant of TBP at position $97^{14}$. With the addition of cuprous ions, reducing agent and hydrogen peroxide, a reactive phenanthroline complex can be formed that is capable of cleaving the DNA in its vicinity. From the cleavage pattern, they quantified the fraction of molecules in the two orientations. For TBP bound to the AdML promoter, $67 \%$ of the proteins bind in the correct orientation and $33 \%$ bind in the opposite orientation. In the presence of TFIIA, the percentage of properly orientated TBP molecules increased to $84 \%$.

A comparison of the results from Schepartz with our measurements in Fig 4. suggests that the peak at 55\% FRET efficiency corresponds to TBP bound to the TATA box in the opposite orientation. Upon longer incubation times or the addition of TFIIA, the subpopulation with a peak at 55\% FRET efficiency decreases. For complexes formed within the flow cell, the ratio of the subpopulation at $40 \%$ FRET efficiency to the $55 \%$ FRET peak is $67 \%$ to $33 \%$, in excellent quantitative agreement with the results from Schepartz.

Additional support for the assignment of the $40 \%$ FRET peak to the correct orientation and the 55\% FRET peak to the opposite orientation of TBP on the AdML promoter comes from comparison with the crystal structure (Fig 1.). The approximate distances between the attachment points for the donor and acceptor molecules is $60 \AA$ for the correct orientation and $\sim 52 \AA$ for the opposite orientation (assuming that the overall structure of the TBP-DNA complex does not change significantly when TBP binds with the opposite orientation). Using a $\mathrm{R}_{0}$ of $59 \AA$ provided by the manufacturer, we estimate the donor-acceptor separation to be $66 \AA$ for TBP in the correct orientation and $58 \AA$ for TBP in the opposite orientation. Considering the fluorophores are attached with long flexible linkers, the agreement is quite good. Even if we ignore the absolute distances, which can be very challenging to determine reliably, the relative donoracceptor separations are in agreement with our hypothesis. The donor-acceptor separation of the complex with TBP in the correct orientation is larger than that of TBP bound in the opposite orientation, consistent with assignment of the $40 \%$ and 55\% FRET subpopulations to TBP bound in the correct and opposite orientations respectively. 


\section{BINDING OF TBP TO THE TATA BOX}

For the measurements presented in Section 3, the FRET signal from the TBP-DNA complex was typically constant. This steady-state FRET signal was achieved only after TBP had firmly bound to the TATA box. Using the spFRET analysis, we are also able to investigate the process of TBP binding onto a promoter region.

\subsection{Results}

To monitor individual binding events of TBP molecules to the TATA box of the AdML promoter, the prism surface was first covered with the DNA probe. A concentration of $500 \mathrm{pM}$ was used to ensure a high surface coverage of DNA for the TBP binding experiments. This concentration would be too high for single molecule experiments on the DNA, but is good for single molecule investigations of the TBP-DNA complexes. Excess DNA, which had not bound to the surface after $10-15$ minutes, was washed away with working buffer. Bind of TBP to the AdML promoter was investigated by flowing a $500 \mathrm{pM}$ TBP solution through the sample chamber. These experiments must be performed with an excess TBP, which leads to a slightly higher background due to freely diffusing TBP molecules in the buffer. Two typical spFRET traces of TBP binding to the DNA are shown in Fig. 5A. Upon TBP binding to the DNA, a FRET signal is immediately observed. The FRET value makes abrupt transitions between different apparently discrete states. To determine whether there are indeed discrete states, a frame-wise histogram was performed. The resulting histogram is shown in Fig. 5B. Six peaks are observable, suggesting that specific discrete states exist.
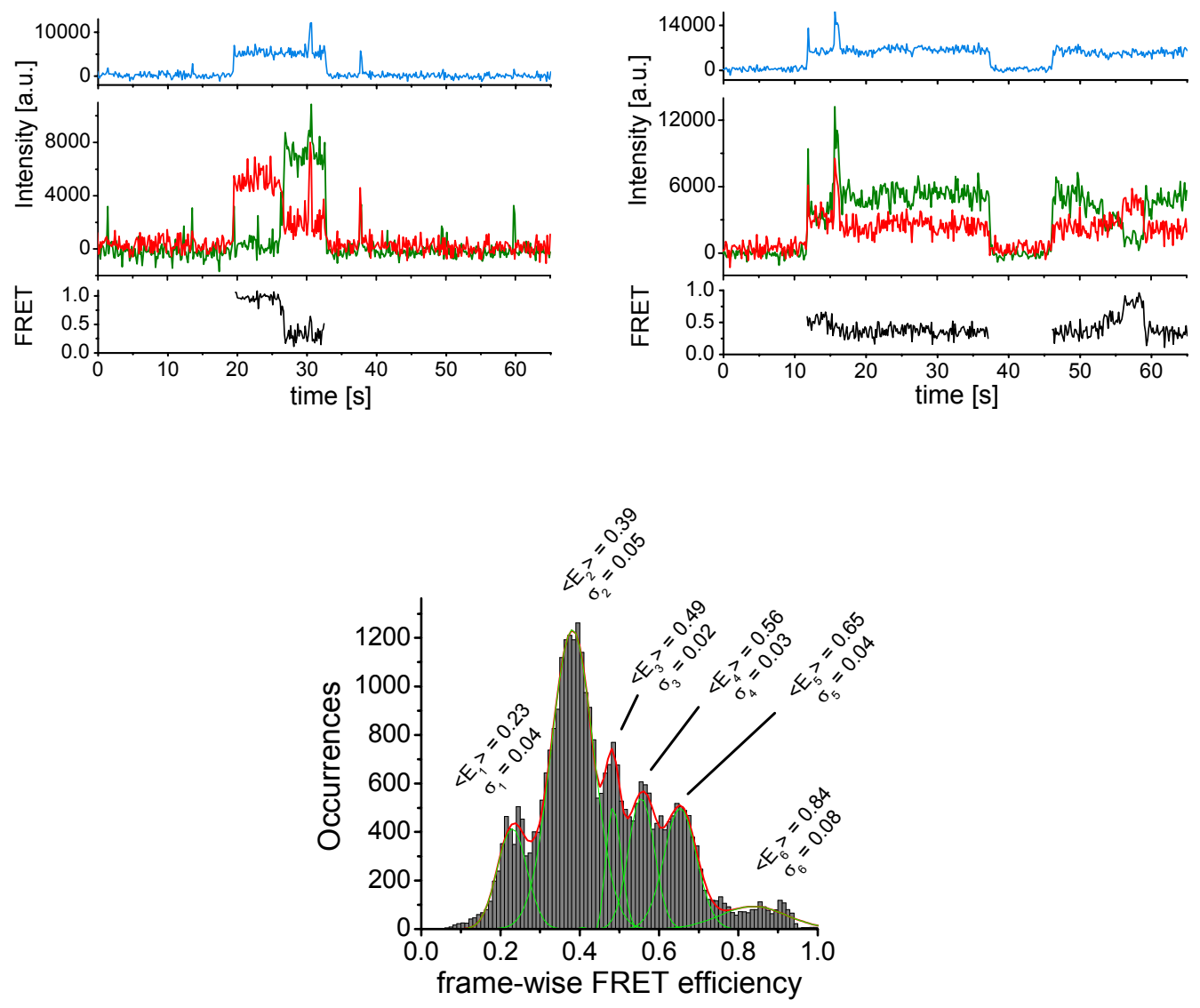

Fig. 5. SpFRET measurements of TBP binding. A) Two spFRET traces recorded after TBP addition showing a dynamic behavior with transitions between different discrete FRET states. B) The resulting frame-wise histogram from the analysis of the FRET steps. At least six different FRET populations are observable. Only steps with a duration longer than $750 \mathrm{~ms}$ and a standard deviation of $<0.1$ were included in the histogram. 


\subsection{Discussion}

Both Parkhurst and Tolić-Nørrelykke have proposed a model for TBP binding to the AdML promoter that includes two intermediate states. What we observe with our spFRET experiments is much more complicated. The frame-wise histogram (Fig. 5B) of the different FRET levels observed in the spFRET measurements shows six different populations. The first step in understanding the dynamics of TBP binding is to identify theses different states. Three of these peaks correspond to what we observed in investigations on the orientation of TBP on the DNA: the peaks at $\sim 20 \%, \sim 40 \%$ and $55 \%$. These states can be assigned to TBP binding at a different location on the DNA, TBP bound in the correct orientation on the TATA box, and TBP in the opposite orientation of the TATA box. The conformations corresponding to the remaining FRET peaks still need to be determined. Once the FRET states have been identified, there is a large amount of information that can be extracted from the spFRET traces. One possibility to extract the kinetic information from this data is to use a Hidden Markov Model (HMM) ${ }^{17}$. From the HMM analysis, we can extract information over the number of states, the FRET level of each state, the transition rates of each state and the transition probabilities between these various states. Such an analysis would provide a detailed insight into the process of TBP binding to the AdML promoter.

\section{CONCLUSIONS}

TBP binding to a promoter site is in important step in the initiation of gene expression in eukaryotic cells. To investigate the mechanism of gene regulation, we developed a single pair Förster Resonance Energy Transfer (spFRET) assay and used it previously to study the impact that negative cofactor 2 (NC2) has upon binding to TBP-DNA complexes. Here, we have applied this assay to investigate the pathway of TBP binding to the AdML promoter site. We verified that TBP binds with two possible orientations on the AdML promoter site. In our experiments, the $40 \%$ FRET peak corresponds to TBP in the correct orientation and the 55\% FRET subpopulation to TBP bound in the opposite orientation. Increased incubation time or addition of TFIIA leads to improved alignment of TBP on the DNA, consistent with what was observed by Schepartz and coworkers ${ }^{14}$. We also applied the spFRET assay to investigate the process of TBP binding to the AdML promoter. The spFRET traces showed multiple FRET states with abrupt transitions between the various states. In contrast to what has been observed by Parkhurst ${ }^{14}$ and Tolić-Nørrelykke ${ }^{9}$, we have detected at least six states during the TBP binding process. The spFRET traces of TBP binding show a rich dynamics and, with the use of a Hidden Markov Model or similar analysis techniques, we hope to extract the kinetic information available from this data and unravel the pathway of TBP binding to the AdML promoter.

\section{REFERENCES}

[1] Schluesche, P., Stelzer, G., Piaia, E., Lamb, D. C. and Meisterernst, M., "NC2 mobilizes TBP on core promoter TATA boxes", Nat Struct Mol Biol, 14(12), 1196-1201 (2007).

[2] Inostroza, J. A., Mermelstein, F. H., Ha, I., Lane, W. S. and Reinberg, D., "Dr1, a TATA-binding proteinassociated phosphoprotein and inhibitor of class II gene transcription", Cell, 70(3), 477-489 (1992).

[3] Meisterernst, M. and Roeder, R. G., "Family of proteins that interact with TFIID and regulate promoter activity", Cell, 67(3), 557-567 (1991).

[4] Nikolov, D. B., Chen, H., Halay, E. D., Hoffman, A., Roeder, R. G. and Burley, S. K., "Crystal structure of a human TATA box-binding protein/TATA element complex", Proc Natl Acad Sci U S A, 93(10), 4862-4867 (1996).

[5] Parkhurst, K. M., Brenowitz, M. and Parkhurst, L. J., "Simultaneous binding and bending of promoter DNA by the TATA binding protein: real time kinetic measurements", Biochemistry, 35(23), 7459-7465 (1996).

[6] Parkhurst, K. M., Richards, R. M., Brenowitz, M. and Parkhurst, L. J., "Intermediate species possessing bent DNA are present along the pathway to formation of a final TBP-TATA complex", Journal of Molecular Biology, 289(5), 13271341 (1999).

[7] Powell, R. M., Parkhurst, K. M., Brenowitz, M. and Parkhurst, L. J., "Marked stepwise differences within a common kinetic mechanism characterize TATA-binding protein interactions with two consensus promoters", Journal of Biological Chemistry, 276(32), 29782-29791 (2001).

[8] Powell, R. M., Parkhurst, K. M. and Parkhurst, L. J., "Comparison of TATA-binding protein recognition of a variant and consensus DNA promoters", Journal of Biological Chemistry, 277(10), 7776-7784 (2002).

[9] Tolić-Nørrelykke, S. F., Rasmussen, M. B., Pavone, F. S., Berg-Sorensen, K. and Oddershede, L. B., "Stepwise bending of DNA by a single TATA-box binding protein", Biophysical Journal, 90(10), 3694-3703 (2006). 
[10] Banik, U., Beechem, J. M., Klebanow, E., Schroeder, S. and Weil, P. A., "Fluorescence-based Analyses of the Effects of Full-length Recombinant TAF130p on the Interaction of TATA Box-binding Protein with TATA Box DNA", J. Biol. Chem., 276(52), 49100-49109 (2001).

[11] Gumbs, O. H., Campbell, A. M. and Weil, P. A., "High-affinity DNA binding by a Motlp-TBP complex: implications for TAF-independent transcription", Embo J, 22(12), 3131-3141 (2003).

[12] Kapanidis, A. N., Laurence, T. A., Lee, N. K., Margeat, E., Kong, X. and Weiss, S., "Alternating-laser excitation of single molecules", Accounts of Chemical Research, 38(7), 523-533 (2005).

[13] Cox, J. M., Hayward, M. M., Sanchez, J. F., Gegnas, L. D., vanderZee, S., Dennis, J. H., Sigler, P. B. and Schepartz, A., "Bidirectional binding of the TATA box binding protein to the TATA box", Proceedings of the National Academy of Sciences of the United States of America, 94(25), 13475-13480 (1997).

[14] Kays, A. R. and Schepartz, A., "Virtually unidirectional binding of TBP to the AdMLP TATA box within the quaternary complex with TFIIA and TFIIB", Chemistry \& Biology, 7(8), 601-610 (2000).

[15] Liu, Y. C. and Schepartz, A., "Kinetic preference for oriented DNA binding by the yeast TATA-binding protein TBP", Biochemistry, 40(21), 6257-6266 (2001).

[16] Wang, Y. and Stumph, W. E., "RNA polymerase II/III transcription specificity determined by TATA box orientation", Proc Natl Acad Sci U S A, 92(19), 8606-8610 (1995).

[17] McKinney, S. A., Joo, C. and Ha, T., "Analysis of single-molecule FRET trajectories using hidden Markov modeling", Biophys J, 91(5), 1941-1951 (2006). 